\title{
Maturation of Cardiorespiratory Interactions in Spontaneous Recovery from Hypoxic Apnea (Autoresuscitation) ${ }^{1}$
}

\author{
WILLIAM M. GERSHAN, MARGARET S. JACOBI, AND BRADLEY T. THACH \\ The Edward Mallinckrodt Department of Pediatrics, Washington University School of Medicine, \\ St. Louis, Missouri 63110
}

\begin{abstract}
The maturation of the process of spontaneous recovery from hypoxic apnea by gasping (autoresuscitation) was characterized in adult, weanling (18-22 d), and infant ( 5 and $10 \mathrm{~d}) \mathrm{BALB} / \mathrm{c}$ mice. ECG and respiration were recorded and hypoxic apnea was induced with $97 \%$ $\mathrm{N}_{2}-3 \% \mathrm{CO}_{2}$. Administration of $21 \% \mathrm{O}_{2}$ at onset of hypoxic apnea resulted in successful autoresuscitation in all 63 animals tested. In all mice, three sequential stages occurred: 1) gasping with marked bradycardia, 2) rapid increase in heart rate (cardiac resuscitation), and 3) increase in respiratory rate (respiratory resuscitation). Despite these similar stages, marked maturational differences were apparent. The times to cardiac and respiratory resuscitation were longer in the pups than in the older mice, whereas the gasp frequency before cardiac resuscitation was lower in the infants. Resuscitation time correlated with gasp interval (1/gasp frequency). In addition, the initial bradycardia at onset of gasping was more pronounced in the pups. A 30- or 60-s delay in oxygen administration after onset of hypoxic apnea caused a marked decrease in the adults' ability to autoresuscitate, without affecting that of the pups. A comparison of gasp frequency in $21 \% \mathrm{O}_{2}$ versus $97 \% \mathrm{~N}_{2}-3 \% \mathrm{CO}_{2}$ showed that the presence of oxygen increased gasp frequency in the pups, but had no effect on gasp frequency in the weanlings or adults. We conclude that autoresuscitation is equally effective in both infant and adult BALB/c mice but only when oxygen is available before the onset of hypoxic gasping. Accordingly, the adult's ability to autoresuscitate rapidly can be viewed as an adaptation required by a limited tolerance for prolonged hypoxia. In contrast, it appears that the infant mouse's greater tolerance of hypoxia allows a slower autoresuscitation with a lower heart rate and gasp frequency. This conserves energy, thus facilitating prolonged survival and enabling recovery when $\mathrm{O}_{2}$ availability is delayed. (Pediatr Res 28: 87-93, 1990)
\end{abstract}

\section{Abbreviations}

HR, heart rate

$R R$, respiratory rate

ANOVA, analysis of variance

$\mathrm{PaO}_{2}$, arterial oxygen tension

Received January 11, 1990; accepted April 2, 1990.

Correspondence: William M. Gershan, M.D., Pulmonary Section, Department of Pediatrics, The Medical College of Wisconsin, Children's Hospital of Wisconsin, 9000 W. Wisconsin Ave., Milwaukee, WI 53201.

Supported by Grant HD 10993-11 from the National Institute of Child Health and Human Development (B.T.T.) and by a Pediatric Pulmonary Research Fellowship Award from the American Lung Association (W.M.G.).

' Presented in part at the Annual Meeting of The Society for Pediatric Research, May 1989
Inability to recover from prolonged apnea has been considered a possible causal factor in the Sudden Infant Death Syndrome. Spontaneous arousal from sleep is one way in which recovery from apnea can occur (1). Recovery from apnea by hypoxic gasping, a process termed "autoresuscitation," is another mechanism (1-4). Autoresuscitation, unlike spontaneous arousal, occurs in the presence of hypoxic CNS depression. Because autoresuscitation occurs after the arousal response has been eliminated by hypoxia, it can be considered a "fail-safe" or "backup" means of ensuring survival.

Although examples of age-related dysfunction of autoresuscitation have been documented by ourselves and others, many basic features of the mechanism, including its normal development, have not been described $(2,5)$. In preliminary studies, we found that BALB/c mice autoresuscitate well at all ages. Therefore, we studied these mice to elucidate the normal maturation of cardiorespiratory interactions that occur in autoresuscitation. Specifically, we wished to determine the relation between age and speed of cardiac and respiratory recovery, degree of bradycardia, and gasping frequency. We also wished to see if delayed oxygenation would alter autoresuscitation ability.

Our findings from these studies indicate maturational adaptations for hypoxic survival: infant mice conserve energy with slow gasping and have the ability to delay recovery until oxygen becomes available, whereas adults have rapid gasping and recovery, but have little tolerance for delays in oxygen availability.

\section{MATERIALS AND METHODS}

Animals. Experiments were performed on BALB/c mice from the local animal facility, Jackson Laboratories (Bar Harbor, ME), and the Charles River Breeding Laboratories (Wilmington, MA). The mice were unrestrained and unanesthetized, except ether anesthesia was used for insertion of ECG electrodes. The induction of hypoxia was deemed ethical because nitrogen inhalation results in rapid loss of consciousness and is recommended as a method of euthanasia for mice by the NIH Guide (6).

Apparatus. In the older mice (weanlings and adults), respiration was recorded using barometric plethysmography, as in previous studies (5). This consisted of a $120-\mathrm{mL}$ jar sealed with a rubber stopper, through which passed an inlet and an outlet tube and a connector to a pressure transducer (Statham pressure gauge PM15E, Hato Rey, Puerto Rico). A slow leak $\left(\mathrm{t}_{1 / 2}=2.0 \mathrm{~s}\right)$ was provided by a 23 -gauge needle to compensate for environmental drifts in temperature and pressure. In the pups ( 5 and $10 \mathrm{~d}$ old), respiration was measured using a loosely fitting latex thoracic balloon connected to a pressure transducer (5). In the infants, warming of the chamber to $32^{\circ} \mathrm{C}$ to maintain a normal oral body temperature of $33 \pm 1^{\circ} \mathrm{C}$ was necessary but prevented use of the plethysmograph, which relies on a temperature gradient between animal and chamber. Anoxia in all animals was produced by flushing the chamber with $97 \% \mathrm{~N}_{2}-3 \% \mathrm{CO}_{2}$. The $\mathrm{CO}_{2}$ concentra- 
tion in the outlet tubing was monitored using a Beckman medical gas analyzer (model LB-2). $\mathrm{CO}_{2}$ was used in the experiments to prevent hypocapnia and also served as a tracer gas to indicate when the chamber was fully flushed with $\mathrm{N}_{2}$.

The ECG was recorded via placement of thoracic, fine wire electrodes (38-gauge Isomid, Belden Co., Chicago, IL) that were connected to a differential AC microelectrode preamplifier (model P15, Grass Instrument Co., Quincy, MA). All signals were recorded on a Beckman R-611 Dynograph pen recorder (Beckman Instruments, Inc., Schiller Park, IL) and also on an FM tape recorder (Hewlett-Packard Co., Palo Alto, CA; model 3968 A) for later playback. Oral temperatures were measured in all 5-, 10-, and 20-d-old mice just before the experiment using a thermistor (Beckman). Oral temperatures were impossible to record in the adult mice, but rectal temperatures were measured (model BAT-12, Sensortek, Inc., Clifton, NJ) in a subsequent group of seven adults studied in an identical manner for temperature data only. Oral temperatures were converted to rectal equivalents by adding $1.9^{\circ} \mathrm{C}$, a difference confirmed by simultaneous measurements of oral and rectal temperatures in six weanlings. This conversion factor assumes that the rectal-oral temperature difference in weanlings is similar to that in the 5- and 10d-old mice in whom rectal temperatures cannot be safely measured.

Protocol. Experiments were performed in infant (5 and $10 \mathrm{~d}$ ), weanling (18-22 d), and adult (older than $40 \mathrm{~d}$ ) mice. Adults were allowed food and water ad libitum. Pups and weanlings remained with their mothers until the time of the experiment. ECG electrodes were inserted $10-15 \mathrm{~min}$ before the anoxic exposure, allowing all animals time to fully recover from the ether.

Three series of experiments were performed. In the first, $21 \%$ oxygen was given at the onset of hypoxic apnea to characterize the cardiorespiratory interactions that occur during successful autoresuscitations in air as well as to examine maturational differences in autoresuscitation ability found previously (5). In the second, oxygen delivery was delayed to determine autoresuscitation ability when $\mathrm{O}_{2}$ availability is not immediate. In the third, nitrogen with $\mathrm{CO}_{2}$ was given instead of oxygen to determine the gasping duration and gasping pattern in anoxia.

Autoresuscitation with early oxygen. Weanling and adult mice were placed in a 120-mL barometric plethysmograph and the 5and 10-d-old pups were fitted with a latex balloon (5) connected to a pressure gauge and then placed in a $60 \mathrm{~mL}$ glass chamber warmed in a water bath. The balloon was placed loosely around the thorax and, therefore, did not impede respiration. The mice seemed unaware of and unaffected by the balloon once it was in place.

At the start of the experiment, the chamber was flushed with air and then humidified $97 \% \mathrm{~N}_{2}-3 \% \mathrm{CO}_{2}$ was flushed in at 12 $\mathrm{L} / \mathrm{min}$ until the $\mathrm{CO}_{2}$ concentration in the outlet tubing reached a plateau of $3 \%$. When the $3 \%$ plateau was reached, the chamber was sealed by closing the inlet tap and clamping the outlet tubing. At the onset of hypoxic (primary) apnea, the outlet tubing was unclamped briefly and oxygen injected into the chamber. Thirty $\mathrm{mL}$ of oxygen was given for the adults, $35 \mathrm{~mL}$ for the weanlings, and $22 \mathrm{~mL}$ for the 5 - and 10-d-old mice. These volumes were previously determined to give an oxygen concentration in the chamber of approximately $21 \%$. Experiments were performed in 20 adult mice, 15 weanlings (aged 18-22 d), 14 10-d-olds (9-11 d), and 14 5-d-olds (4-6 d).

Autoresuscitation with delayed oxygen. These experiments were the same as the autoresuscitation with early oxygen experiments, except that $\mathrm{O}_{2}$ was given either 30 or $60 \mathrm{~s}$ after hypoxic apnea occurred. The volumes of oxygen used were the same as those used in the air experiments. Experiments with a 30 -s delay were performed in five adults and five pups (aged 5-10 d) and with a 60-s delay in four adults and six pups (aged 7-10 d).

Attempted autoresuscitation in anoxia. These experiments were the same as the autoresuscitation with early oxygen exper- iments, except that instead of oxygen, 97\% $\mathrm{N}_{2}-3 \% \quad \mathrm{CO}_{2}$ was injected into the chamber at the onset of hypoxic apnea. Experiments were performed in nine adults, six weanlings (aged $20 \mathrm{~d}$ ), and five pups (aged 7-9 d).

Analysis of results. Cardiac and respiratory resuscitation times were defined as the time from the first gasp after hypoxic apnea to the return to $60 \%$ of control HR and RR, sustained for at least $3 \mathrm{~s}$. We used a percentage of baseline rate as an index rather than an absolute number because the baseline HR and RR varied somewhat in the four age groups. In addition, an index such as this was necessary because although the onset of eupnea was usually well defined, the increase in RR was occasionally more gradual. The gasp duration was the time from the first to the last gasp. The last gasp was defined as the last gasp to appear on the pen chart.

Statistics. Comparisons of cardiorespiratory parameters among the four age groups were made using analysis of variance and unpaired $t$ tests employing the Bonferroni correction for multiple comparisons where appropriate. Resuscitation times and gasp frequencies were compared using linear regression analysis. Differences in survival in air and with delayed oxygen were assessed using the Fisher's exact test. A $p$ value of $\leq 0.05$ was considered significant.

\section{RESULTS}

Autoresuscitation with early oxygen. Table 1 shows baseline physiologic parameters in the four age groups. All 63 animals in this experiment successfully autoresuscitated from hypoxic apnea. In all mice, three sequential stages occurred: 1 ) gasping with marked bradycardia, 2) cardiac resuscitation (rapid increase in HR to $>60 \%$ of baseline), and 3) respiratory resuscitation (increase in RR to $>60 \%$ of baseline). The sequence of autoresuscitation in an adult mouse is illustrated in Figure $1 a$ and that of an infant mouse (age $5 \mathrm{~d}$ ) in Figure $1 b$ and $c$.

Although all mice successfully recovered, there were several striking maturational differences in the pattern in which autoresuscitation occurred. As shown in Figure 2, the cardiac resuscitation time ( $p<0.05$, Bonferroni comparison) and the respiratory resuscitation time ( $p<0.01$, Bonferroni comparison) were longer in the infant mice than in the older mice. In addition, the time interval between cardiac resuscitation and respiratory resuscitation was longest in the 5 -d-old mice and became progressively shorter with increasing age.

The number of gasps after oxygen that were required before cardiac resuscitation occurred was similar in the age groups, ranging from 2.8 gasps in the 5 -d-olds to 4.3 gasps in the adults. However, gasp frequency during this time was higher in older animals and lower in young animals ( $p<0.01$, ANOVA), as shown in Figure 3.

Of particular interest was the observation that a higher gasp frequency was related to a faster autoresuscitation as demonstrated in Figure 4, a plot of respiratory resuscitation time versus 1 /gasp frequency (the interval between gasps; $r=0.747, p<$ $0.01)$.

In all animals during hypoxic apnea, profound sinus brady-

Table 1. Age and baseline physiologic parameters in early oxygen studies*

\begin{tabular}{lcccc}
\hline \multicolumn{1}{c}{ Group } & Age $(\mathrm{d})$ & $\mathrm{HR} / \mathrm{min}$ & $\mathrm{RR} / \mathrm{min}$ & $\mathrm{Temp}_{\mathrm{r}}\left({ }^{\circ} \mathrm{C}\right) \dagger$ \\
\hline 5-d-old pups & $4.8 \pm 0.6$ & $506 \pm 47$ & $200 \pm 48$ & $34.2 \pm 1.3$ \\
10-d-old pups & $10.0 \pm 0.9$ & $603 \pm 46$ & $232 \pm 45$ & $34.8 \pm 1.4$ \\
Weanlings & $20.1 \pm 1.7$ & $661 \pm 62$ & $327 \pm 75$ & $35.5 \pm 0.8$ \\
Adults & $117.2 \pm 33.5$ & $626 \pm 95$ & $353 \pm 75$ & $(33.6 \pm 0.9) \ddagger$ \\
\hline
\end{tabular}

* Data are shown as mean $\pm \mathrm{SD}$.

$\dagger$ Rectal temperatures are shown. Oral temperatures in 5-, 10-, and 20-d-old mice were converted to rectal equivalents by adding $1.9^{\circ} \mathrm{C}$.

$\$$ Rectal temperatures measured in a group of seven adults studied in an identical manner to the original adults. 
a.

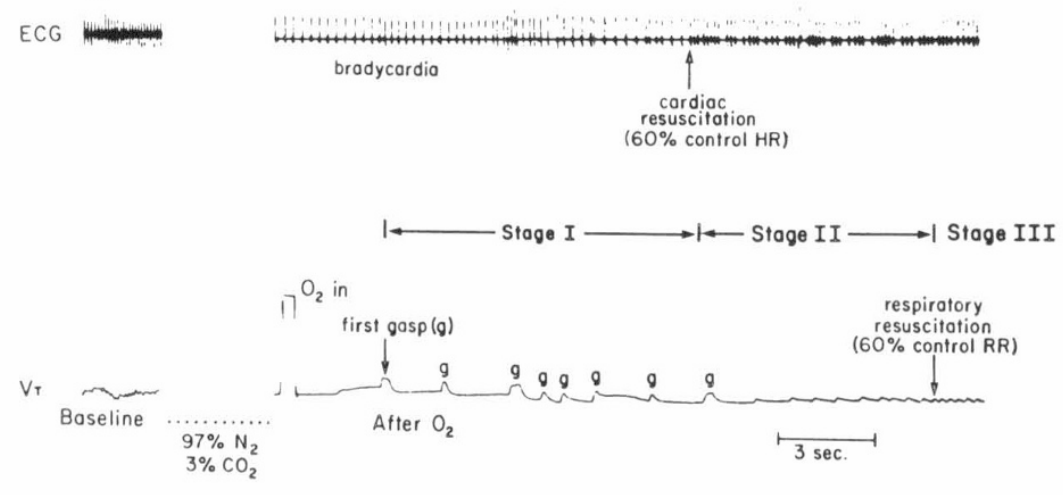

b.
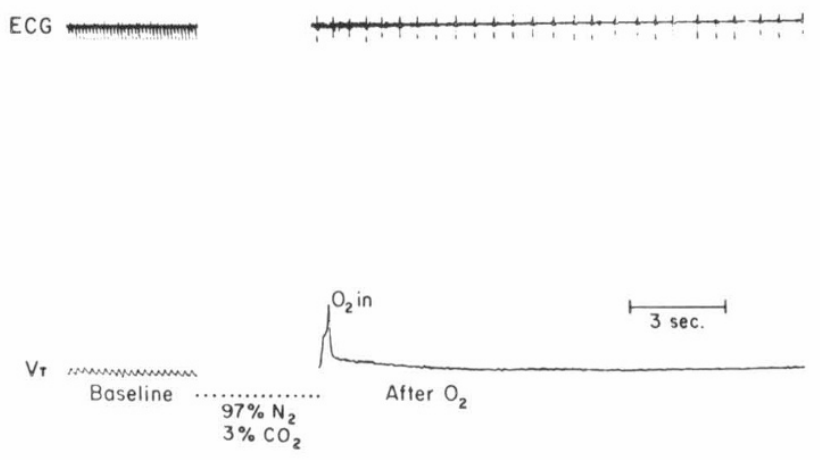

C.
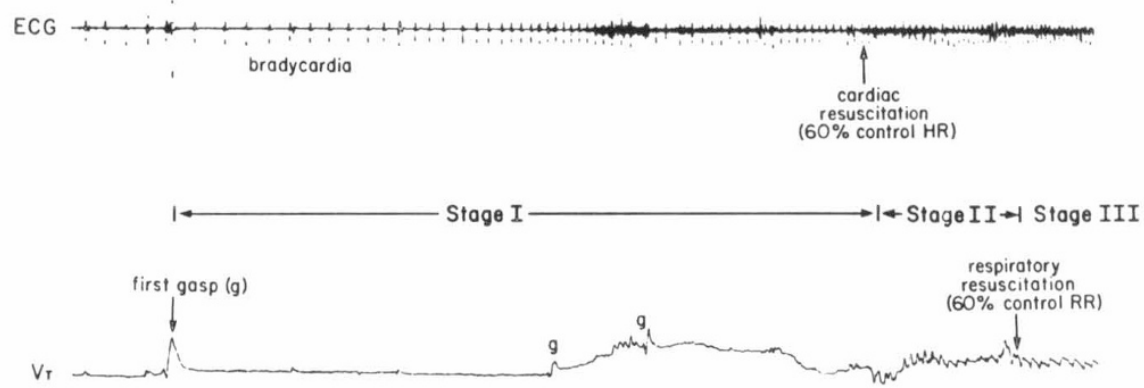

Fig. 1. Autoresuscitation sequence in an adult mouse $(a)$ and in a 5-d-old mouse ( $b$ with continuation on $c$ ). Tracings include ECG and respiration, which is recorded using a barometric plethysmograph in the adult and a thoracic balloon in the pup. Baseline measurements and the period after oxygen administration are shown. Times at which gasps $(g)$, cardiac resuscitation (HR $>60 \%$ baseline), and respiratory resuscitation (RR $>60 \%$ baseline) occurred are indicated. Stages $I, I I$, and $I I I$ refer to the periods of gasping with marked bradycardia, the onset of cardiac resuscitation, and the onset of respiratory resuscitation, respectively.

cardia developed, and in approximately $50 \%$ of the animals in each age group, nodal bradycardia occurred for several seconds. Sinus and nodal bradycardias usually converted to normal sinus rhythm coincident with cardiac resuscitation. HR during hypoxic apnea is shown in Figure $5 a$. There was a significantly lower HR in both the 5- and 10-d-old mice compared with the two older groups ( $p<0.005$, Bonferroni comparison). Because the baseline HR in the 5-d-olds was lower than in the three other age groups (Table 1), the percentage of baseline HR at hypoxic apnea was also compared (Figure $5 b$ ). In this case, the age difference persisted. With the exception of 2 nd degree heart block, which briefly occurred in two adults, no other arrhythmias were seen in the mice.

Autoresuscitation with delayed oxygen. A delay in oxygen availability resulted in a significant difference in the autoresuscitation ability of the adults versus the infants ( $p<0.05$; Fig. 6). Six of the nine adults subjected to delayed oxygen failed to autoresuscitate. Of the six, five did not gasp after oxygen was given and one gasped only once after oxygen. The only infant that failed to autoresuscitate gasped after oxygen, and cardiac resuscitation followed. However, 2nd degree heart block ensued, eupnea never occurred, and the animal eventually died.

Attempted autoresuscitation in anoxia. Gasping duration in nitrogen was heavily dependent on age. The mean gasping duration was $24 \mathrm{~s}$ in nine adults, $30 \mathrm{~s}$ in six weanlings, and $548 \mathrm{~s}$ in five infants $(p<0.01$, ANOVA). The mean number of gasps during this time was 17 in the adults, 14 in the weanlings, and 34 in the infants $(p<0.01$, ANOVA). Figure 7 displays a comparison of instantaneous gasp frequency (1st three gasps) for animals given oxygen or nitrogen. In adults, gasp frequency was essentially constant and did not change after oxygen administration. In infants, however, gasp frequency remained extremely low in nitrogen, but increased progressively when oxygen was given $(p<0.01$, ANOVA). Gasp frequency for weanlings in nitrogen (1st gasp, $28.5 \pm 7.1$ gasps/min, mean \pm SEM) was between that of infants and adults, and like adults, was not significantly affected by oxygen $(p>0.05)$. To eliminate the possible effects of cardiac resuscitation on gasp frequency after 


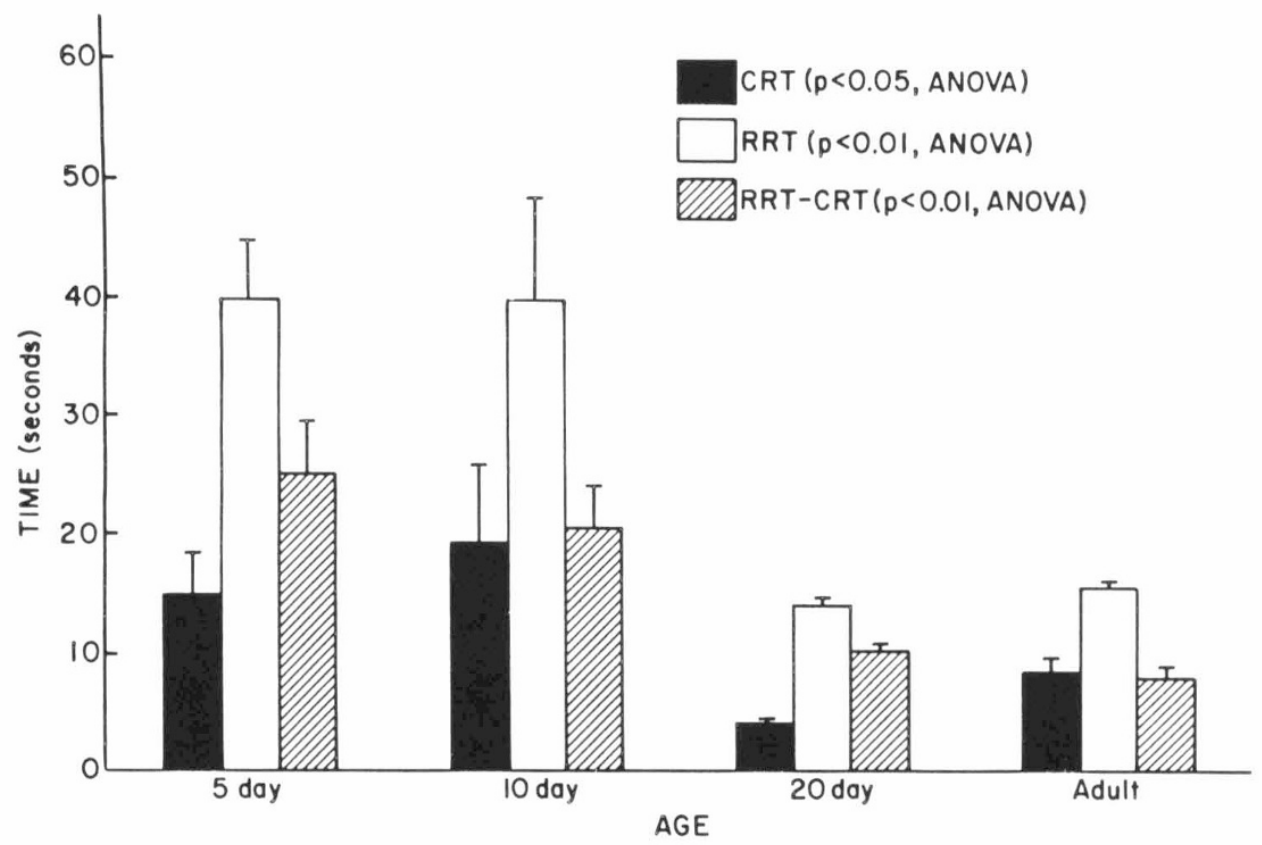

Fig. 2. Cardiac $(C R T)$ and respiratory $(R R T)$ resuscitation times in the four age groups. In addition, the time interval between CRT and RRT (RRT-CRT) is shown. Error bars, SEM.

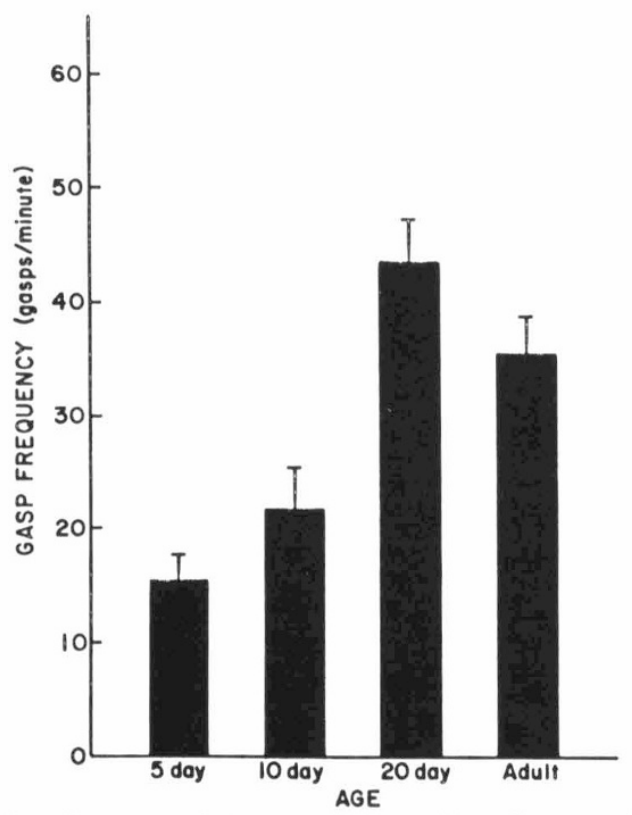

Fig. 3. Gasp frequency before occurrence of cardiac resuscitation in the four age groups. The increase in gasp frequency with maturation was statistically significant $(p<0.01$, ANOVA $)$. Error bars, SEM.

oxygen administration, we excluded from analysis all gasps occurring just before and after cardiac resuscitation. The increase in gasp frequency after oxygen was given to the infants persisted $(p<0.05$, ANOVA), whereas the gasp frequency in the adults and the weanlings did not change.

\section{DISCUSSION}

We have found that the mechanism that causes autoresuscitation is very robust in BALB/c mice of different ages, inasmuch as all 63 mice successfully recovered. Thus, the autoresuscitation defect resulting in inability to recover from hypoxia previously found in weanlings of two related strains of mice (SWR and SW) was absent in BALB/c mice (5). Although the underlying factor that accounts for these differences is unclear, we believe that genetic influences are a major factor because environmental and

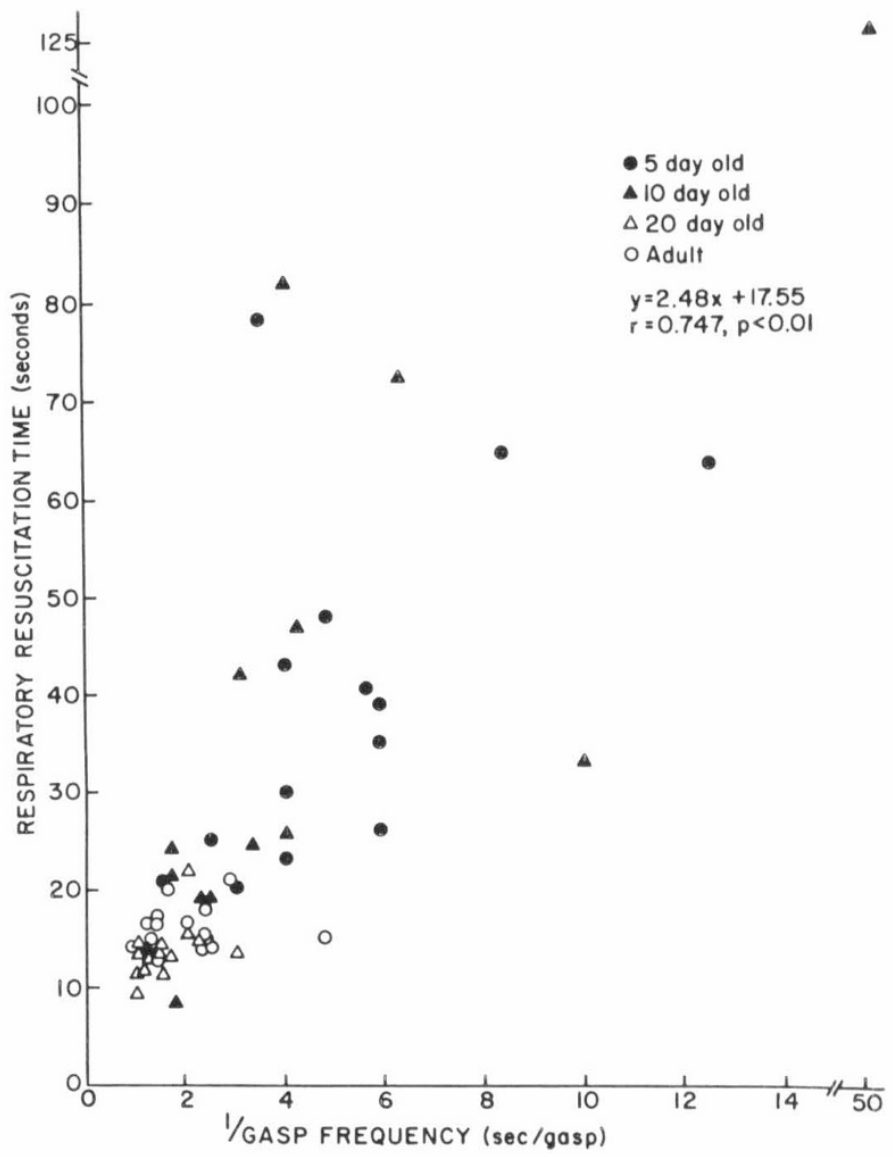

Fig. 4. Comparison of respiratory resuscitation time to 1/gasp frequency (interval between gasps) in all mice. Each symbol represents an individual mouse. Significant relationship among all mice is shown. Significant relationship remained $(p<0.01)$ after exclusion of outlier in right upper margin.

test conditions for the different mice were similar. However, it seems that these interstrain differences in autoresuscitation ability may be quantitative rather than qualitative, inasmuch as we 

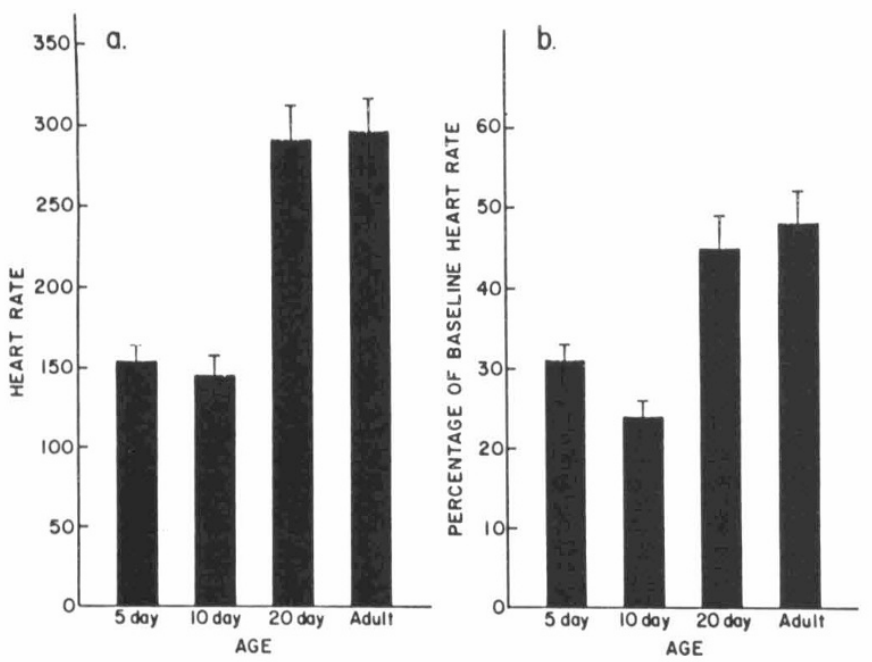

Fig. 5. HR at onset of hypoxic apnea in the four age groups. a) HR $(p<0.01$, ANOVA $) ; b)$ percentage of baseline $\mathrm{HR}(p<0.01$, ANOVA $)$. Error bars, SEM.

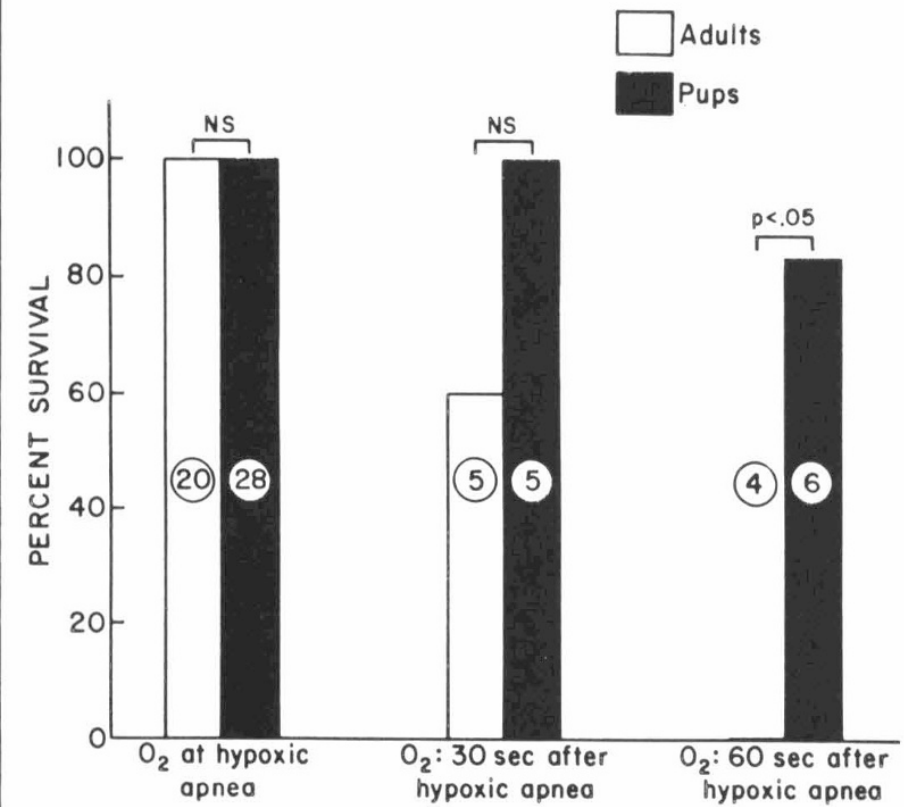

Fig. 6. Percentage survival in pups and adults when oxygen was given at hypoxic apnea onset, and 30 and $60 \mathrm{~s}$ after onset. Numbers of animals in each group are shown.

have previously shown that a similar autoresuscitation defect can be induced in BALB/c mice by repeated anoxic exposures (7).

We used two different techniques for recording respiration because the need for warming the younger mice prevented use of the plethysmograph. Both techniques have been validated in previous studies (5). However, it is possible that nonrespiratory movements could have been mistaken for gasps using the balloon technique in the younger mice. From past studies in which such movements were evaluated, we estimate that this error would have been infrequent. However, due to this possible error, gasp frequency may have been slightly overestimated in the 5- and 10 -d-old mice. If this were the case, the true differences in gasp frequency between infants and adults would have been even larger than those reported.

We observed that cardiorespiratory events occurred in three sequential stages in successful autoresuscitation from hypoxic apnea. Others have observed the increase in HR after inhalation of air (8) or the recovery of eupnea after gasping (2); however, the sequence of these events and the invariable occurrence of HR recovery before that of eupnea has not been noted previously.
A similar situation has been observed, however, in artificial resuscitation from hypoxic apnea. Campbell et al. (9) used positive pressure lung inflations to resuscitate asphyxiated rabbits and found that the first sign of successful autoresuscitation from severe anoxia was an increase in HR. Similarly, Godfrey (10) noted an increase in $\mathrm{HR}$ and blood pressure before respiration in artificially resuscitated newborn rabbits. Other studies have shown that the duration of gasping activity in complete anoxia is lengthened by effects of the circulation (11). Thus, maintenance of cardiovascular activity during anoxia appears to sustain the respiratory centers. Our studies suggest that respiratory recovery may be dependent on cardiac or circulatory recovery and may not occur until a critical level of brain perfusion is reached.

Although infant BALB/c mice routinely autoresuscitate from hypoxic apnea, we have found that they do so much more slowly than do older animals; this sluggishness was reflected in both cardiac and respiratory resuscitation times. In addition, once cardiac resuscitation had occurred, the delay time to respiratory resuscitation was longest in the 5 -d-old mice and progressively shortened with increasing age. Although temperature is known to affect gasping duration in anoxia (12), we do not believe that it could account for the maturational difference in autoresuscitation time. We verified this by comparing baseline temperature in the mice to both cardiac and respiratory resuscitation times and found no correlation ( $r=0.089$ and -0.053 , respectively). Autoresuscitation speed may be related to an individual's oxygen stores at the time that gasping commences. However, Guntheroth and Kawabori (2) found that during progressive hypoxia, the $\mathrm{PaO}_{2}$ threshold level below which gasping began was very similar in both infant and adult monkeys. Although we were unable to measure $\mathrm{PaO}_{2}$ in our study, it seems likely that a similar situation would exist in the mice. Previous studies have attributed the newborn's increased resistance to anoxia to a lower cerebral metabolic rate compared with that of the adult $(13,14)$. We speculate that this low metabolic rate may also result in a generalized slowing of the components of autoresuscitation, thereby causing longer resuscitation times.

We did, in fact, find that a low gasp frequency was associated with a prolonged recovery. In contrast, higher gasp frequencies conceivably reoxygenate the heart and respiratory centers more rapidly, thus hastening recovery. Previous studies have found that newborns of various species are capable of gasping much longer during anoxia than adults $(15,16)$, but we are unaware of any similar studies comparing gasp frequency. It has been found that preadministration of glucose, which results in increased substrates for glycolysis during anoxia, also causes reduced gasp frequency (17). The basis for this effect on gasp frequency is unknown; however, newborn mammals are known to have greater glycogen stores than adults, and this could be a basis for their reduced gasp frequency (18). In any case, reduced gasp frequency would result in reduced energy expenditure during anoxia and therefore can be viewed as a mechanism for prolonging anoxic survival in the younger animals.

Gasp frequency in infant mice abruptly increased once oxygen became available. We wondered if cardiac resuscitation could have played a role in this effect. However, when the influence of cardiac resuscitation on gasp frequency was eliminated, the oxygen effect persisted, implying that oxygen, by itself, is a regulator of gasp frequency. It has been shown that gasping does not occur during the course of progressive hypoxia until a certain threshold value of $\mathrm{PO}_{2}$ is reached (2), and also that gasping onset is independent of input from peripheral chemoreceptors (19). However, neither of these reports cite effects on gasp frequency. St. John and Knuth (20) studied gasping produced by brainstem lesions in carotid body-denervated cats. They found that reduction of $\mathrm{PaO}_{2}$ from normoxia to hypoxia caused a transient increase in gasp frequency. Although this model is different in a number of ways from our own, it does suggest that gasp frequency can be influenced by $\mathrm{PaO}_{2}$ independent of the peripheral chemoreceptors. If the speed of autoresuscitation is influenced by gasp 


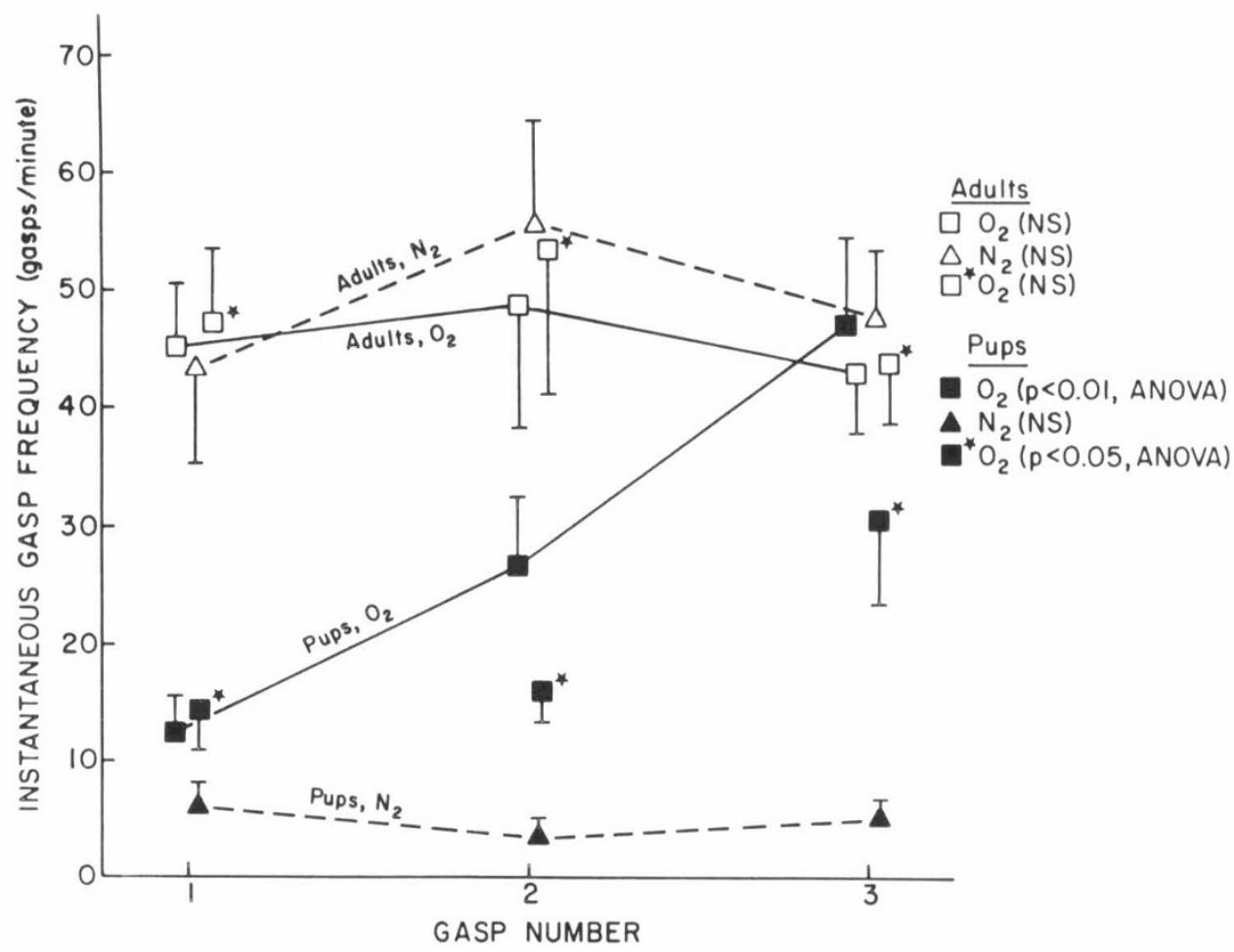

Fig. 7. Instantaneous gasp frequency $(1 /$ intergasp interval $\times 60)$ in oxygen and in nitrogen (comparing the 1st three gasps after hypoxic apnea). For this comparison, 5- and 10-d-old mice were similar and, therefore, were grouped together as pups. The asterisks refer to gasp frequency in oxygen after elimination of the possible effects of cardiac resuscitation. See text for discussion. Error bars, SEM.

frequency as our findings suggest, then it clearly would be advantageous for the animal to have positive feedback regulation of gasping in which oxygen availability increases gasp frequency and thereby speeds resuscitation. Why such regulation was not apparent in the older animals is unclear, but such an effect might be masked by their already rapid baseline gasping rate.

With onset of hypoxic apnea, we observed a greater degree of sinus bradycardia in the young mice. Several studies have shown that the infant's heart beats much longer in anoxia than the adult's $(15,21)$. Moreover, the infant's heart contains more cardiac glycogen than the adult's (18), a factor that has been directly related to the heart's capacity to beat for prolonged periods in total anoxia (22). Accordingly, it has been suggested that bradycardia may promote anoxic survival by decreasing cardiac oxygen and glycogen consumption $(23,24)$. Of relevance is the observation of Dawes et al. (25) that the depletion of cardiac carbohydrate during anoxia is less rapid in lambs than in adult sheep. Although HR was not compared in that study, it is possible that a greater degree of bradycardia in the lambs played a role in their increased conservation of cardiac carbohydrate. Similarly, the greater degree of bradycardia during anoxia in our young mice is possibly evidence of a protective mechanism that conserves cardiac glycogen, thereby prolonging survival in anoxia.

Our studies have shown that the autoresuscitation mechanism is as effective in the adult as it is in the mouse pup only if oxygen is available promptly after onset of hypoxic apnea. We found that delays in providing $\mathrm{O}_{2}$ were generally well tolerated by the young mice but resulted in frequent autoresuscitation failure in adults. Our findings indicate that when $\mathrm{O}_{2}$ availability is delayed, autoresuscitation may fail either because gasping is ineffectual or because gasps do not occur. The latter mechanism could have been predicted from the gasping duration in nitrogen, but the former mechanism is novel and suggests that early gasps may be more effective in reoxygenation than later gasps. The mechanism whereby gasps are rendered ineffectual is unclear. Given that the ability to autoresuscitate diminishes as duration of anoxia in- creases, it is functionally appropriate that the speed of autoresuscitation increases with maturation, as tolerance of prolonged anoxia decreases. Otherwise, prolonged autoresuscitation attempts in the adult might prove ineffectual even when $\mathrm{O}_{2}$ is available. Maturation, therefore, entails a shift from a prolonged anoxic survival time, facilitated by cardiorespiratory mechanisms associated with slow autoresuscitation, to a relatively brief anoxic survival time associated with rapid autoresuscitation. If these processes were to mature at disparate rates-for example, if the transition from slow to rapid autoresuscitation lagged behind the shift to a shorter anoxic survival time-then it is possible that intermediate-aged mice might develop an inability to autoresuscitate. In fact, such altered patterns of development may underlie the autoresuscitaion defect that occurs transiently during development in SWR mice (5).

In regards to fatal clinical apnea, our data suggest that forms of apnea associated with delays in oxygen availability (e.g. delayed or ineffectual gasping) might have increased mortality as patients mature, because ability to autoresuscitate would worsen. However, as we have only studied two strains of mice, each with different maturational patterns in ability to autoresuscitate, it is difficult to predict the pattern that might be expected in other species.

\section{REFERENCES}

1. Hunt CE, Brouilette RT 1987 Sudden infant death syndrome: 1987 perspective. J Pediatr 110:669-678

2. Guntheroth WG, Kawabori I 1975 Hypoxic apnea and gasping. J Clin Invest $56: 1371-1377$

3. Fewell JE, Kondo CS, Dascalu V, Filyk SC 1989 Influence of carotid denervation on the arousal and cardiopumonary response to rapidly developing hypoxemia in lambs. Pediatr Res 25:473-477

4. Willinger M 1989 SIDS: a challenge. Journal of NIH Research 1:73-80

5. Jacobi MS, Thach BT 1989 Effect of maturation on spontaneous recovery from hypoxic apnea by gasping. J Appl Physiol 66:2384-2390

6. Committee on Care and Use of Laboratory Animals, Institute of Laboratory Animal Resources, Commission on Life Sciences, National Resource Council 1980 National Institutes of Health, Bethesda, MD (NIH publication no. 80-23) 
7. Gershan WM, Jacobi MS, Thach BT 1990 Spontaneous recovery from hypoxic apnea: a comparison of adept and inept mice. Am Rev Respir Dis 141:812(abstr)

8. Adolph EF 1969 Regulations during survival without oxygen in infant mammals. Respir Physiol 7:356-368

9. Campbell AGM, Cross KW, Dawes GS, Hyman AI 1966 A comparison of air and $\mathrm{O}_{2}$, in a hyperbaric chamber or by positive pressure ventilation, in the resuscitation of newborn rabbits. J Pediatr 68:153-163

10. Godfrey S 1968 Respiratory and cardiovascular changes during asphyxia and resuscitation of foetal and newborn rabbits. Q J Exp Physiol 53:97-118

11. Himwich HE, Bernstein AO, Herrlich H, Chesler A, Fazekas JF 1942 Mechanisms for the maintenance of life in the newborn during anoxia. Am J Physiol 135:387-391

12. Adolph EF 1948 Tolerance to cold and anoxia in infant rats. Am J Physiol 55:366-377

13. Holowach-Thurston J. McDougal DB 1969 Effect of ischemia on metabolism of the brain of the newborn mouse. Am J Physiol 216:348-352

14. Duffy TE, Kohle SJ, Vannucci RC 1975 Carbohydrate and energy metabolism in perinatal rat brain: relation to survival in anoxia. J Neurochem 24:271276

15. Selle WA, Witten TA 1941 Survival of the respiratory (gasping) mechanism in young animals subjected to anoxia. Proc Soc Exp Biol Med 47:495-497

16. Glass HG, Snyder FF, Webster E 1944 The rate of decline in resistance to anoxia of rabbits, dogs, and guinea pigs from the onset of viability to adult life. Am J Physiol 140:609-615

17. Holowach-Thurston J, Hauhart RE, Jones EM 1974 Anoxia in mice: reduced glucose in brain with normal or elevated glucose in plasma and increased survival after glucose treatment. Pediatr Res 8:238-243

18. Shelley HJ 1961 Glycogen reserves and their changes at birth and in anoxia Br Med Bull 17:137-143

19. Woodrum DE, Parer JT, Wennberg RP, Hodson WA 1972 Chemoreceptor response in initiation of breathing in the fetal lamb. J Appl Physiol 33:120 125

20. St. John WM, Knuth KV 1981 A characterization of the respiratory pattern of gasping. J Appl Physiol 50:984-993

21. DeHaan RL, Field J 1959 Anoxic endurance of cardiac and respiratory function in the adult and infant rat. Am J Physiol 197:445-448

22. Stafford A, Weatherall JAC 1960 The survival of young rats in nitrogen. J Physiol (Lond) 153:457-472

23. Swann HG, Christian JJ, Hamilton C 1954 The process of anoxic death in newborn pups. Surg Gynecol Obstet 99:5-8

24. Adolph EF. Hoy PA 1960 Ventilation of lungs in infant and adult rats and its response to hypoxia. J Appl Physiol 15:1075-1086

25. Dawes GS, Mott JC, Shelley HJ 1959 The importance of cardiac glycogen for the maintenance of life in foetal lambs and newborn animals during anoxia. J Physiol (Lond) 146:516-538 